\title{
PARALLEL OBJECT CLASSIFICATION ALGORITHMS IN IMAGES
}

\author{
Luong Chi Mai
}

Viện Công nghệ thông tin

\section{Introduction}

Image processing and recognition belong to those research areas of artificial intelligence where computers contribute significantly to solving problems which have been only in the competence of the human intelligence. There are several serious demands which lead to the present interest at parallel computers for solving these problems. Huge amount of data to be processed require computers with high operational and memory capacity, as well as reliability. Due to their ability to fulfill these conditions, parallel processors seem to become dominating for future trends in solving image processing, pattern recognition and other related artificial intelligence problems.

Our attention is devoted to the problem of object classification in digital images. Well known statistical approaches were chosen to be modified for parallel implementation. The computational regime under consideration is SIMD (Single Instruction Multiple Data). From this perspective three methods will be examined and parallelized for the SIMD-type computer developed and operated at the Institute of Technical Cybernetics in Bratislava [4]. We restrict ourselves here only to a brief description of its main features.

Computations are performed in parallel modules which are composed of the MDA (Multidimensional Access Memory) block, the arithmetic-logical unit (ALU) and of the permutation network (PN). The MDA memory block consits of $256 \times 256$ bit subblocks (pages). Data are located in memory fields to which there exits access in both vertical and horizontal directions as well as in their combinations. To each word of the memory one 1-bit processor element of the ALU is assigned. This unit 
contains also registers of the length 256. One of them serves as a mask register where respectively 1 and 0 at a given position indicates that word in the memory where the result of an operation is or is not written in. The permutation network allows data permutations according to the application under cosideration. Most frequently used are 2 -type permutations, $k=0,1, \ldots, 7$. The control memory contains the programm for the parallel machine. For the data transfer from the control memory to the MDA memory one 32-bit register is at user's disposal.

The standard functions are realized in a form of macroinstructions and procedures. The application programs from the image processing are described in [2]. This paper brings description of new application algorithms related to the parallel object classification in images. The approaches under parallelization are the Bayes classification, the K-mean and the ensemble average classifier. The Bayes algorithm for the SIMD-type parallel machine is presented in the section 2 of this contribution. It is based on the evaluation of mean vectors and covariance matrices. The automatic K-mean classifier is formulated in the section 3. From the point of view of parallel realization, the most effective seems to be the ensemble average classifier which is described in the concluding section.

We note that the algorithms presented are under programming realization in a parallel assember programming language. Results of computational experiments will be published elsewhere.

\section{The Bayes classifier}

Let $x$ be a vector (pixel) in the $N$-dimensional Euclidian space $E$. For a set of $K$ vector classes $C=\left\{C_{i}, i=1,2, \ldots, K^{*}\right\}$, let $P\left(C_{i}\right)$ be a priori class-probability of $C_{i}, f\left(x \mid C_{i}\right)$ be class-conditional probability density function and $P\left(C_{i} \mid x\right)$ be the conditional probability that $x$ belongs to the class $C_{i}$.

The Bayes strategy mininuzes the misclassification error and it is based on the minimization of the function [5]

$$
L_{X}\left(C_{i}\right)=\sum_{j=1}^{K^{-}} P\left(C_{j} \mid x\right)=1-P\left(C_{i} \mid x\right)
$$

Let the set of decision functions is defined by

$$
G_{i}(x)=f\left(x \mid C_{i}\right) P\left(C_{i}\right), \quad i=1,2, \ldots, K
$$

According to (1) and the relation of propotionality between $P\left(C_{i} \mid x\right)$ and $f\left(x \mid C_{i}\right) P\left(C_{i}\right)$, an unknown vector $x$ is classified into the class $C_{i}$ if and only if

$$
i G_{i}(x)=\max _{j}\left[G_{j}(x)\right] \text { for } j=1,2, \ldots, K
$$


Assume that each $f\left(x \mid C_{j}\right), j=1,2, \ldots, K$ is $N$-dimensional Gaussian density function with mean vector $\mu_{j}$ and covariance matrix $R_{j}$. Considering $P\left(C_{1}\right)=P\left(C_{2}\right)=\ldots=$ $P\left(C_{K}\right)$, we obtain [5],

$$
G(x)=-\frac{1}{2}\left(\ln \left|R_{j}\right|+\left(x-\mu_{j}\right)^{T} R_{j}^{-1}\left(x-\mu_{j}\right)\right),
$$

where $|R|$ denotes the determinant of $R_{j}$ and $R_{j}^{-1}$ is the inverse matrix of $R_{j}$.

In practice, mean vector and covariance matrix of ęach class must be evaluated from training sets of vectors belonging to them. For a given class $C_{j}, j=1,2, \ldots, K$ formulas for evaluating respectively the elements $\mu_{j}^{k}, k=1,2, \ldots, N$ of $m_{j}$ and $\delta_{j}^{i k}, i, k=$ $1,2, \ldots, N$ of $R_{j}$, are

$$
\mu_{j}^{k}=\frac{1}{S_{j}} \sum_{l=1}^{s_{j}} y_{k}^{l}
$$

and

$$
\delta_{j}^{i k}=\frac{1}{S_{j}} \sum_{l=1}^{S_{j}}\left(y_{i}^{l}-\mu_{j}^{i}\right)\left(y_{k}^{l}-\mu_{j}^{k}\right)
$$

In (4) and (5), $y_{i}^{l}$ and $y_{k}^{l}$ denote respectively $i^{\text {th }}$ and $k^{\text {th }}$ component of th training set vector y belonging to $C_{j} . S_{j}$ is the total number of training set elements in $C_{j}$.

$\begin{array}{lllll}\text { 1st } & y_{1}^{1} & \ldots \ldots & y_{1}^{M} & \begin{array}{l}\text { Mth training } \\ \text { vector of }\end{array} \\ \text { training } & y_{2}^{1} & \ldots \ldots & y_{2}^{M} & \text { class } C_{1} \\ \text { of class } & \vdots & \ldots \ldots & \vdots & \\ C_{1} & y_{N}^{1} & \ldots \ldots & y_{N}^{M} & \\ & \vdots & \ldots \ldots & \vdots & \\ 1 \text { st } & y_{1}^{1} & \ldots \ldots & y_{1}^{M} & \text { Mth training } \\ \text { training } & y_{2}^{1} & \ldots \ldots & y_{2}^{M} & \text { vector of } \\ \text { of class } & \vdots & \ldots \ldots & \vdots & \text { class } C_{1} \\ C_{1} & y_{N}^{1} & \ldots \ldots & y_{N}^{M} & \\ & 0 & \ldots \ldots & 0 & \end{array}$

Fig.1. The illustration of the training vector location.

In the parallel Bayes algorithm for the parallel associative computer of the SIMDtype, data elements represent $\mathrm{N}$-dimensional vectors. Assume that each component possesses one of 256 gray-level values ( 8 bits) correspondingly to the gray-level scale of the image. It means that one page of the MDA memory can be divided into $M$ 
column fields $(M=32) F_{1}, F_{2}, \ldots, F_{M}$. Assume that the page of the memory is divided into 4 horizontal blocks, which consist of 64 rows each. These blocks are filled-up in a such manner that all 1st dimension components of the trainig set vectors belonging to the 1st class are located in the first row, the 2 nd dimension components of the training set vectors of the 1st class are located in the 2 nd row, etc. To store all elements in training sets for all classes, $K^{*} * N$ rows of each block are reserved. Free remaining rows of each block should be filled-up by zeros. The location of data in the 1st horizontal memory subblock (64 rows) shows Fig.1. Remaining $S_{j}-M$ training vectors for each $j=1,2, \ldots, K$ are located in the same way in next horizontal block of the memory page. If the number of elements in training sets for all classes is larger than the memory page size, additional pages of the MDA memory can be used.

Except the fields for storing the training set elements, the algorithm requires to reserve also some other auxiliary fields in the MDA nemory. Field $\mathrm{H}$ is used to store mean vectors and fields $B_{1}, B_{2}, \ldots, B_{N}$ are reserved for the elements of the covariance matrices of all classes. These fields are also divided horizontally into 4 blocks of 64 rows each. In ith row, $i=1,2, \ldots, N$ of each subblock in $H$ there is located a mean value corresponding to ith dimension. The field $B_{1}$ is used for storing elements $\delta_{11}, \delta_{22}, \ldots, \delta_{N N}$ of the covariance matrix, the field $B_{2}$ for $\delta_{12}, \delta_{23}, \ldots, \delta_{N-1, N}$ etc., while the field $B_{n}$ contains $\delta_{1 N}$. The illustraton of these fields is shown on Fig.2.

$\begin{array}{llllll} & \mu_{1}^{1} & \sigma_{1}^{12} & \sigma_{1}^{12} & \ldots & \sigma_{1}^{1 N} \\ & \mu_{1}^{2} & \sigma_{1}^{22} & \sigma_{1}^{23} & \ldots & 0 \\ \text { Class } C_{1} & \mu_{1}^{N-1} & \sigma_{1}^{N-1, N-1} & \sigma_{1}^{N-1, N} & \ldots & \ldots \\ \vdots & \mu_{1}^{N} & \sigma_{1}^{N N} & 0 & \ldots & 0 \\ & \vdots & \vdots & \vdots & \vdots & \vdots \\ & & & & & \\ \text { Class } C_{K} & \mu_{1}^{1} & \sigma_{1}^{12} & \sigma_{1}^{12} & \ldots & \sigma_{1}^{1 N} \\ & \mu_{1}^{2} & \sigma_{1}^{22} & \sigma_{1}^{23} & \ldots & 0 \\ & \mu_{1}^{N-1} & \sigma_{1}^{N-1, N-1} & \sigma_{1}^{N-1, N} & \ldots & \cdots \\ & \mu_{1}^{N} & \sigma_{1}^{N N} & 0 & \ldots & 0 \\ & 0 & 0 & 0 & 0 & 0 \\ & H & B_{1} & B_{2} & & B_{N}\end{array}$

Fig. 2. The illustration of the contents of the fields $H, B_{1}, B_{2}, \ldots, B_{N}$ in one memory block of 64 rows.

For calculation of all components of mean vectors by the formula. (4), blockpermutations to add-up corresponding values in each dimension for all classes are used in the parallel algorithm. The result is stored in the field $H$. 
The algorithm uses two auxiliary fields $H_{1}, H_{2}$ divided also into 4 blocks. In $H_{1}$ there is accumulated the sum $\sum_{i=1}^{M}\left(F_{j}-H\right)$.

Both fields are used to apply block-permutations by addition of corresponding partial sums from (5). The result of multiplication of $H_{1}$ and $H_{2}$ are diagonal elements of the covariance matrices which are stored in the field $B_{1}$. Covariance matrix elements in the fields $B_{2}, B_{3}, \ldots, B_{N}$ are evaluated using the operation of acyclical shift in each subblock and the field multipllication. In last $K$ steps the division of each $B_{1}, B_{2}, \ldots, B_{N}$ by the number of elements in the corresponding training set is performed.

In the parallel Bayes classification phase, iuput data represent image pixels stored in the MDA memory in the line interleaved manner,i.e. the first row of the first dimension is located in the first row of the memory page, in the second row of the page there is located the first row of the second dimension,etc., in the kth row there is the first row of the $k$ th dimension, $k=3,4, \ldots, N$. If each pixel possesses one of 256 gray-level values and the number of dimensions cquals to $N=4$ then in one page of the MDA memory could be stored one image of the size $32 \times 64$. Besides the pixel values, input data for the classification phase are mean vectors and covariance matrices of all classes. These vectors and matrices are results from the learning phase and they are stored in an external storage derice. The field $\mathrm{H}$ is divided into blocks of size $N$, where $N$ is the dimension number. The first block is used to store $\mathrm{N}$-dimensional mean vectors for the first class,etc., the kth block stores the mean vector for $k$ th class, $k=2,3, \ldots, K$. Output of the classification is an image where a pixel represents that class label, to which the corresponding pixel of the original image belongs.

The algorithm consists of two parts, according to the calculation of formula (2) and (3) respectively. Main problem in evaluating the formula (3) is the calculation of the inverse matrix and determinant of each covariance matrix $R_{j}, j=1,2, \ldots, K$. The algorithm uses a modification of the parallel subroutine JORDAN for solving linear systems of algebraic equations by mean of the Gause-Jordan elimination method with column piroting [3]. The final operation is to find maximal values according to the formula (2). It is realized in parallel hy comparing all columns.

\section{Parallel automatic $\kappa$-mean classifier}

This type of clssifier does not require any assumption on probability distribution of pattern vectors. The knowledge available is that there is a sequence of vectors $x_{1}, x_{2}, \ldots, x_{N}$ from $N$-dimensional euclidian space. It is assumed that $N<<n$. Without making use of training samples, the nethod classifies a sequence of vectors $x_{1}, x_{2}, \ldots, r_{N}$ into $K$ classes $C_{1}, C_{2}, \ldots, C_{K}$,

The automatic $K$-mean algorithm can be formulated in following steps [1] : 
Step 1: Choosing $K$ initial centres $z_{1}(1), z_{2}(1) \ldots, z_{K}(1)$ of all elasses. This selection is done more or less arbitrarily.

Step 2: In $k^{\text {th }}$ iteration step, vecturs $x_{l}, l=1,2, \ldots, n$ may be distributed into the classes $C_{1}, C_{2}, \ldots, C_{K}$ by applying the rule

$$
x_{l} \in C_{j}(k) \Longleftrightarrow\left\|x_{l}-z_{i}(k)\right\|<\|x-z(k)\|
$$

for all $j=1,2 \ldots, k ; i \neq k$.

In (6), $\|$.$\| denotes the euclidian norm, C_{j}(k)$ represents the set of vectors entering the class with the centre $z j(k)$ and index $k$ denotes the iteration number. In the case of equality in (6), $x_{l}$ can be classified either to the class $C_{i}$ or to that class $C_{j}$ for which the equality holds.

Step 3: Based on the result of step 2, new centres $z_{1}(k+1), z_{2}(k+1), \ldots, z_{K}(k+1)$ of all classes must be selected minimizing the function

$$
J_{j}=\sum_{x \in C_{j}(k)}\left\|x-z_{j}(k+1)\right\|, j=1,2, \ldots, K .
$$

It is well known that the minimum $z_{j}(k+1)$ of $(7)$ occurs in the average point of all vectors contained in the set $C_{j}(k), j=1,2, \ldots, K$. It is evaluated by

$$
z(k+1)=\frac{1}{N_{j}} \sum_{x \in C_{j}(k)} x
$$

where $N_{j}$ is the number of rectors in $C_{j}\left(k^{\prime}\right)$.

Stcp 4: If

$$
\|z(k+1)-z(k)\|<\varepsilon \text { for all } j=1,2 \ldots, K
$$

$(\varepsilon>0$ is chosen $)$, then the algorithm terminates. In the opposite case, repeat from the step 2 .

A convergence of the $K$-mean algorithm depends on a rather large number of factors. In most cases the application of algorithm requires some testing steps concerning the proper value of the parameter $\mathrm{K}$ and the initial allocation of the centres of classes., In the parallel $K$-mean algorithm for the SIMD-type computer, it is considered that the multilevel image is stored in $M$ fields $F_{1}, F_{2}, \ldots, F_{M}$ of the MDA memory; where the size of each column $F_{i}, i=1,2, \ldots, M$ depends on the gray-level scale of pixels. It is assummed a multilevel inage with the gray-level scale from 0 to 255 , i.e. 8 bits must be used to represent onc pixel in the memory and in this case $M=32$. Each $N$-dimensional pixel is stored in the MDA memory in the interleaved form (Fig.3). For simplicity of illustration let $K=8, N=4$. Assume that each pixel $x$ and each centre $z$ of class $C_{j}, j=1,2, \ldots, K$ are represented in a vector form,i.e. $x=\left(x_{l 1}, x_{l 2}, \ldots, x_{l N}\right)$ and $z=\left(z_{j 1}, z_{j 2}, \ldots, z_{j N}\right)$ respectively (see Fig.3), where number $q=64 *(M-1)$. 


$\begin{array}{lllll}x_{11} & \ldots \ldots & x_{q-63.1} & z_{11} & \text { Centre } \\ x_{12} & \ldots \ldots & x_{q-63.2} & z_{12} & \text { for } \\ \vdots & \ldots \ldots & \vdots & \vdots & \text { class } \\ x_{1 N} & \ldots \ldots & x_{q-63 . N} & Z_{1 N} & C_{1} \\ \vdots & \ldots \ldots & \vdots & \ldots & \vdots \\ \vdots & \ldots \ldots & \vdots & z_{k 1} & \ldots \\ x_{64.1} & \ldots \ldots & x_{q 1} & z_{k 1} & \text { Centre } \\ x_{64.2} & \ldots \ldots & x_{q 2} & \cdots & \text { for } \\ \vdots & \ldots \ldots & \vdots & z_{k N} & \text { class } \\ x_{64 . N} & \ldots \ldots & x_{q N} & 0 & C_{1} \\ F_{1} & \ldots \ldots & F_{M} & Z & \end{array}$

Fig.3. The illustration of image fields and field for the centres of classes.

A column $z$ with the width of 32 bits is provided for calculating and storing new centres in each iteration. During the evaluation of the algorithm, one boolean matrix $M$ of $K=8$ columns $M_{1}, M_{2}, \ldots, M_{K}$ (1 bit per column) and one matrix $L$ of $M$ columns $L_{1}, L_{2}, \ldots, L_{M}$ ( 3 bits per column) are needed for intermediate labeling of classes. Field $H$ plays the role of histogram of class labels. The output is a matrix located in the MDA memory with elements possessing values from 1 to 8 correspondigly to that class label which is assigned to a given pixel by the classification. The algorithm is divided into following main parts according to steps formulated above:

(i) Selection of $K$ initial centres of all classes. The first $K * N$ rows in the first column $F_{1}$ are selected as $K$ initial centres and stored in the field $z$.

(ii) Pixels $x$ located in the fields $F_{1}, F_{2}, \ldots, F_{M}$ are distributed into $K$ classes by the rule $(6)$.

a. Calculation of $x-z_{j}(k)$ for $j=1,2, \ldots, K$.

b. Calculation of the norm $\|x-z(k)\|$ for $j=1,2, \ldots, k$ using block-permutations with permutation factors 2 and 2 (since $N=4$ ). The results of substeps a. and b. are stored in auxiliary memory fields $x z_{1}, x z_{2}, \ldots, x z_{K}$.

c. Finding maximal values in fields $x x_{1}, x z_{2}, \ldots, x z_{K}$. Label corresponding to these values will be stored in the $M$ columns $L_{1}, L_{2}, \ldots, L_{M}$.

(iii) Calculation of new centres of all classes $C_{j}, j=1,2, \ldots, K$ by (7). In a field $\mathrm{H}$ the number of pixels in all classes is stored. During evaluation of the histogram vector $\mathrm{H}$, boolean columns $M_{1}, M_{2}, \ldots, M_{K}$ are used for marking those positions in $L_{1}, L_{2}, \ldots, L_{M}$ where the same class label occurs.

(iv) Test of convergence according to (9). 


\section{Ensemble average classifier}

As it was the case in the previous classifier, no assumption is made on the probability distribution or density function of the pattern vectors. The only knowledge provided is a sequence of $K$ sets of training pattern vectors with a known classification. The number of vectors in each class $C_{j}, j=1,2, \ldots, K$ must be greater than the dimension of the feature space $N$. Classification of an unknown pattern vector $\mathrm{x}$ into one of the classes is carried out as follows [7]:

Step 1: Estimate the average pattern vector for each class by

$$
m_{j}=\frac{1}{S_{j}} \sum_{l=1}^{S_{j}} x_{l}, j=1,2, \ldots, K,
$$

where $S_{j}$ is the number of training pattern vectors in $C_{j}$ and $x_{j}, l=1,2, \ldots, S_{j}$ are the pattern vectors belonging to $C_{j}$.

Step 2: An unknown pattern vector $\mathrm{x}$ is classified into a class $C_{j}$ iff

$$
\left[\left(x-m_{i}\right)^{T}\left(m_{i}-m_{J}\right)\right]^{2} \geq\left[\left(x-m_{j}\right)\left(m_{i}-m_{j}\right)\right]^{2}
$$

$\mathrm{i} \mathrm{i} \mathrm{j} \mathrm{j} \mathrm{i} \mathrm{j} \mathrm{for} \mathrm{all} i, j=1,2, \ldots, K ; j \neq i$.

After $(k-1)$ comparisons in step 2, the algorithm classifies an unknown vector $\mathrm{x}$ into the correct class.

Ensemble average classification algorithm for a parallel SIMD-type computer possesses as input an image stored in the MDA memory by columns in the interleaved form (as in the K-mean algorithm described previously). Let $K$ mean vectors of the training sets be stored in the fields $H_{1}, H_{2}, \ldots, H_{K}$ of the MDA memory. $H$ is a field containing $K 1$-bit columns. In each row of this field, there is only one value 1 corresponding to the label of that class, which is assigned to a given pixel by the classification. For $\mathrm{K}=8$, an example of the field $\mathrm{H}$ is shown in the following table:

\begin{tabular}{|c|c|c|c|c|c|c|c|c|}
\hline$H_{j} / H$ & $H_{1}$ & $H_{2}$ & $H_{3}$ & $H_{4}$ & $H_{5}$ & $H_{6}$ & $H_{7}$ & $H_{8}$ \\
2 & 0 & 1 & 0 & 0 & 0 & 0 & 0 & 0 \\
7 & 0 & 0 & 0 & 0 & 0 & 0 & 1 & 0 \\
4 & 0 & 0 & 0 & 1 & 0 & 0 & 0 & 0 \\
1 & 1 & 0 & 0 & 0 & 0 & 0 & 0 & 0 \\
8 & 0 & 0 & 0 & 0 & 0 & 0 & 0 & 1 \\
\hline
\end{tabular}

Allocation of the image field and mean vectors are shown in Fig $4 \mathrm{a}$ and Fig. $4 \mathrm{~b}$ respectively, where number $q=64 *(M-1)$ in Fig.4a. For simplicity of representation let us consider $N=4, K=8$ and 256 gray-levels of image pixels. 
The parallel ensemble average classification algorithm is very simple. It is divided into 3 parts. The first part is the calculation of the formula (11) by using only vector subtraction, multiplication and comparision operations. The second one consists in forming the vectors $H_{1}, H_{2}, \ldots, H_{K}$ by using masked vector operations. As a result we have the field $H$ where in each word there is only one bit possessing the value 1 . The third part of the algorithm consists in the histogram calculation from $H_{1}, \dot{H}_{2}, \ldots, H_{K}$. It is realized by the technique for parallel evaluation of histogram on the parallel associative computer given in [6].

$\begin{array}{lll}x_{11} & \ldots \ldots & x_{q-63.1} \\ x_{12} & \ldots \ldots & x_{q-63.2} \\ \vdots & \ldots \ldots & \vdots \\ x_{1 N} & \ldots \ldots & x_{q-63 . N} \\ \vdots & \ldots \ldots & \vdots \\ x_{64.1} & \ldots \ldots & x_{q 1} \\ x_{64.2} & \ldots \ldots & x_{q 2} \\ \vdots & \ldots \ldots & \vdots \\ x_{64 . N} & \ldots \ldots & x_{q N} \\ F_{1} & \ldots \ldots & F_{M}\end{array}$

Fig.4a. Image allocation

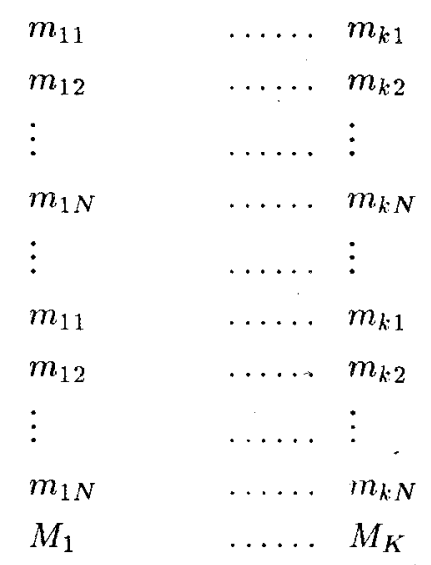

Fig.4b. Mean vectoc allocation

\section{References}

1. Gonsales, Dv. Tu, Principy raspoznawaniq obrazow. Moskwa, Mir 1978, str. 5056.

2. Lucka M., Muchova M. and Vajtersic M., Image processing algorithms for parallel system of the PPS-SIMD type. Sis- temy obabotki znanij i izobravenij. T.1. Smolenice 1986, 157-162.

3. Majlingova O. and Vajtersic M., Jordanova metoda riesenia systemov linearnych rovnic na paralelnom asociativnom pocitaci. Vyskumna sprava UTK SAV, Bratis - lava, 1988.

4. Richter K.et al., The architecture of PPS-SIMD. Sistemy obrabotki znanij i izobravenij. T.2, Smolenice 1986, 51-59.

5. Swain P.H., Image data alysis in remote sensing. Digital Image Processing, Vol.5, No.4, 1979, 200-235.

6. Vajtersic M. and Giang Vu Thang : Threshold and histogram algorithms for a parallel associative computer. Computers and Artificial Intelligence, Vol.5, No.2, $1986,143-161$. 
7. Zaki F.W., Abd el-Fattah A.I., Enab Y.M. and Konyaly S.H., An ensemble average classifier for pattern recognition machines. Pattern Recognition, Vol.21, No.4, $1988,327-332$.

\section{Abstract}

The contribution concerns a parallelization of object classification algorithms for a SIMD-type parallel machine. It is assummed that gray-level values of image pixels are located in the orthogonal memory block on which a vector of one-bit processor operates in the bit-serial and word-parallel mode. For this computer, the Bayes classification algorithm, the $K$-mean algorithm and the ensemble average classifier are described. 